5. 5 .

Die hinder, Enkel und Nachliommen des verstorbenen Dr. Kudolph Brandes sollen, sofern sie unbemiltell sein sollten, bei der Darreichung dieses Stipendiums den Vorzug geniessen.

S. 6.

Dic Verleihung für einen Zögling kann auf nicht länger als höchstens drei Jahre gereicht werden.

\$. 7.

Ausser den Brandes'schen Kindern, Enkeln und Nachlommen haben alle Sölne der Mlitglieder und Ehrenmitglieder des Vereins, welche dazu beigetragen haben, sowie später der folgenden Mitglieder und Ehrennitglieder, Anspruch auf dieses Stipendium, sofern deren Vermügensverhältnisse die Unterstütznng winnschenswerth machen sollien. \$. 8 .

Die Verleihung geschieht durch das Directorium des Apothekervereins in der jährlichen Generalversammlung.

$$
\text { S. } 9 .
$$

Wer dasselbe erhalten will, hat sich unter Ueberreichung des Gelurtszeugnisses, sowie Schul - und Universitäts -, oder sonstige Zeugnisse, im Juni jeden Jahrs an den zeitigen Oberdirector des Vereins zu wenden.

\title{
\$. 10.
}

Ungünstige Zeugnisse oder unsittliclier Lebenswandel verwirken die Entziehung des Stipendiums.

\section{2) Medicinal - Gesetzgebungs - Angelegenheiten.}

Das Wesen der Pharmacie und die zeitgemässen Mittel zu dessen Verbesserung; ein Schriftchen von Gustav G $a r b e$, conditionirendem Apotheker in Stettin. Erörtert rom Apotheker Schlot feldt in Oschersleben.

Der IIr. Verfasser lat sich das Motto gewälit:

Die Drangsal alle soll ich offenbaren,

Dic ich gesehn und meistens selbst erfahren?

Wer sich die Aufgabe stcllt, die Calanitäten und Drangsale eines Faches öffentlich vor der Welt zu proclamiren und sich als Reformator desselben anzukündigen, der muss sich im Besitz umfassender Erfahrungen wähnen; vorzüglich aber den Beruf und die Fähigheiten habejn, ohne Uebertreibung der Wahrheit gemäss, seine Schilderungen darzulegen.

Was nun zuerst den Beruf zur Herausgabe dieser Schrift betriff, so konnte Hr. Garbe sich nur an den alten Denkspruch erinnern : ,Was Deines Amtes nicht ist, da lasse Deinen Vorwitz" - dann wäre der Verf. zu der Einsicht gelangt, dass er als ein Unberufener weiser handelie, wenn er die vorgeschlagenen Reformen den vom Staate dazu als anerkannt qualificirten Mämuern überlassen hätte, die zu seiner Zeit schon die besten Nittel und Wege zur Ausglejchung der obwaltenden Verhältnisse finden werden, ohne den Hrn. Garbe als Wegweiser zu bedürfen.

In Bezug der dem Verf. eigenen Fähigkeiten, um die Heformen dieses grossen Werkes auszuführen. müssen wir allerdings das Bekenntniss ablegen, Jass derselbe in seincr Broschüre viele Geistesfähigkei- 
ten, besonders aher die Aulagen gezeigt hat, mit cminenter Beredisamkeit alle Zustände der l'barmacie in einem vergrösserten Maassstabe und unwahren lichle darzustellen.

IIr. Garbe erzählt in der Einleitung zuerst der Wahrheit gemäss, welchen Einfluss die mit der Pharmacie verbundenen Wissenschaften auf so viele Zweige der bürgerlichen Beschaftigungen zum Wohl der Menschheit und vorzngsweise zur Erhaltung des Lehens ausgeübt haben; derselbe gesteht es ein, dass eine grosse Anzahl berühmter Naturforscher aus der Schule der Plarmacie hervorgegangen ist, worunter namentlich die sich im Gebiete der Chemie Ausgebildeten den ersten Rang einnehmen. Der Verf. erzählt uns nun aber, dass sich die Verhältnisse leider in der uenesten Zeit ganz anders gestaltet und es dahin gekommen ist, dass die l'harmacie an dem Rande eines Abgrundes stehe, in welchen sie unfehlbar versinken werde, wenn ihr nicht der Staat durch eine energische Maassregel zu Ilïlfe komme.

Hr. Garbe hat sich die Aufgabe gestellt, in seiner Schrift die Beweise zu führen für diese Behauptung, und die Mittel anzudeuten, durch welche den jetzt bestehenden und immer mehr überhand nehmenden Uebelständen gründlich abgeholfen werden könne. Zunächst erklärt der Verf., wie in den letzter Decennien so viele Menschen von beschränkten Kenntnissen und untergeordnetem Verstande nur um den täglichen Verdienst arbeiteten, um aus ihren Officinen cinen möglichst grossen Gewinn zu ziehen; wesshalb denn vorzugsweise reiche Leule ihre Kinder, sofern sie von beschräukten Gieistesfähigkeiten waren, um in irgend einem andern Stande ihr Glick zu machen, das Apothekerfach erlernen liessen, um binnen 8 oder 10 Jahren in Besitz einer Apotheke zu gelangen. Durch die angenommene Ausicht des Publicums, dass eine Apothelie ein wahres, ,Rittergut"6 sei, entstand der verderbliche Ilandel mit den Privilegien, in Folge dessen durch schnöden Wucher, der damit getrieben, dem Fortschritt der Wissenschaften feindich entgegengetreten wurde. Das Talent, sagt lIr. Garbe, vermag sich nicht mehr Bahn zu brechen; Geld isi der einzige Hebel, und hebt derselbe als merkwürdig hervor, dass seit $20-25$ Jahren kein Einziger, der seit dieser Zeit der Apothekerkörperschaft einverleibt wurde, es zu einer Auszeichnung gebracht hat, um daran denken zu können, neben den hundert berühmten Männern der vergangenen Jahrhuuderte, dic aus der Pharmacie hervorgegangen sind, einen Plaiz zu verdienen? Nan wird finden, sagt der Verf., dass die grosse Mehrzahl in einem Zeitraum von $20-30$ Jahren bereits einen Rückschritt von mehreren Jahrhunderten genacht hat, weil damals die Apotheker ihre Medicamente selbst bereiteten, während jetzl die meisten Apotheken blosse Dispensiranstatten sind u. s. w.

Dic übertriebenen und in Extreme übergehende Darlegungen dieser Sachverhältnisse sind so augenfällig, als dass diese noch einer Entgegnung bedürften. Der Verf. mag, wenn er will, mit dem Geschicke hadern und es "Schichsalsiücke" nennen, wenn ihm die Mittel nicht zu Gebote stehen, um eine Apotheke zu acquiriren. Es ist diess von jeher nit allen Verhältnissen des Lehens so gewesen, dass die Söhne wohlhabender Eltern früher selbstständig wurden und zum Eigenthum gelangten, als diejenigen, denen diese Uebel fehlen. Ilieran ist aber: das als Aushängeschild bezeichnete ,Apothekerfach" nicht schuld. Die ausgezeichnetsten Talente müsseu sich of kümmerlich ihren Unterhait erwerben, während weniger Befähigle vom Glücke besser bedacht wurden und angenehmer leben können. 
Die Behauplung des Verf., dass die Mehrzahl der Pharmaceuten einen Rïckschritt von mehreren Jahrhunderten gemacht hat und die Pharmacie überhaupt an den Abgrunde des Verderbens steht, worin sic unfehlbar versinken muss, ist eben so unwahr. Wir hatten noch im Jai d. J. Golegenheit, in Berlin aus dem Ilunde eines hochgeachteten und berühmten Gelehrten die Versichertung zu hören, dass sich die Pharmacie eben im 19. Jalırhundert zu ciner solchen ehrenvollen Stufe herangebildet habe, dass die Ansprüche, die in Betreff des Wissens an den jungen Pharmacenten gemacht werden, viel bedeutender geworden wärcn, in Folge dessen aus dem Apothekerfach, und namentlich in Gebiete der Chemie, viele wackere junge Leute hervorgegangen, so dass, wenn die berühmten Chemiker Deutschlands im Vergleich zu dencı Englands und Frankreichs aufgezählt würden, sich die Zahl wie 9 zu 1 herausstellte. Den Angaben dieses ausgezeichneten Gelelirten können wir unbedingt Glanhen schenken, da derselbe mit vieleo Ländern in wissenschaftlicher Berührung steht und auch bei der Ober-Examinationscommission in Berlin fungirt, mithin das reifste Urtheil abzulegen vermag, ob dic jetzigen jungen Pharmaceuten in der Ausbildung so plötalich un diverse Jahrhunderte zurückgekommen sind und das Staatsexamen ableiern, wie sich der Verf. ausgedrückt hat.

Ir. G a r be geht non, nachdem er bewiesen hat, dass es in dem Apothekerstande dem Reichthum gelungen ist, das Talent von einer Bahn zu drïngen, die ihm angewiesen ist, zu den gewöhnlichen Klagen, zu dem schnöden Wucher, über, der mit den Privilegien getrieben worden ist, wodurch der Staat veranlasst wurde, keine Privilegien mehr, sondern Concessionen, Personalgerechtigkeiten zu ertheilen. Es gelang aber, sagt der Verf., durch die verderblichsten Klugheitsnanivyres den concessionirten Apothekern, ihre Apotheken zu eben so enormen Preisen zu versteigern, wie für die Privilegirten gezahlt warden, wodurch denn endlich der Slaat gezwungen ward, durch eime Ministerialverfügung vom 13. August v. J. dem Wucher mil Personalgerechligkeiten dahin Grenzen zu sctzen, dass es fernerhin allein den königl. Kegieruagen gestallet sein soll, dem geeignetsten Bewerber die erledigte Concession zu übertragen, und zwvar für das Inventarium. Zunächst recensirt derselbe die in der Beilage der Cölnischen Zeitung vom 14. April d. J. erschienene Schrift, und belehrt den Unkundigen, dass darin kein Unterschied zwischen Concessionen und Privilegien genacht sei.

Was den Wucher betriff, den sich leider durch den oftern Verkauf und Wiederankauf der privilegirten Apothekell in den letzten Decennien mehre unserer Collegen zu Schulden kommen liessen, so ist die Wahrheit des Hervortretens nicht abzuläugnen, und wahrscheinlich diese auch allein der Grund der neuern Verordnungen! Uebrigens hat es oftmals mit dem verschrienen ,Wucher" eine ganz curiose Bewandniss; davon hat uns jüngst $H r$. College $R$ itz in $W$ esel eine treffende Definition im Archiv der Pharmacio rom Septbr. S. 343 mitgetheilt, die sehr zur Beachtung zu empfehlen ist.

Wenn der Verf., der in der Cölner Zcitung eine Auseinandersctzung des Sachverhältnisses lieferte, keinen Unterschied zwischen Concessionen und Privilegien gemacht haben soll, so kann sich diess nur aus der Ansicht und der Meinung des Verf. erklären lassen, da die diesseits der Elbe gelegenen Apotheken sich in Besitz von Privilegien-Documenten befinden, die aber durch einen Machtspruch während der westphälischen Fremdherrschaft im Jahre 1810 beim Erschei- 
nen des Gewerbesteucrgesctzes aufgehoben wurden. Aber Se. Hochselige Königl. Majestät Friedrich Wilhelm III. erliess die spätern Verordnungen, wodurch alle diese benannten Apotheken den privilegirten fast in allen Verhältnissen gleichgestellt werden sollten, so dass die Vererbung an die Söhne und Schwiegersöhne, wie hei den privilegirten, genehmigt wurde. Beim Verkauf dieser Apotheken mussten indess die königl. Regierungen die Einwilligung geben, die auch nie versagt worden ist, im Gegentheil ist der l'reis von den königl. Regierungen in vielen Fällen (wovon die Beweise vorliegen) sogar ausdrücklich bestimmt und festgeseizt. Die von den IIrn. Garbe hiermit bezeichneten Apotheken sind daher lieineswegs neu angelegte, geschenkte, sondern theuer erkaufte Apotheken, ähnlich den privilegirlen, und bein Verkauf nach dem bisherigen jährlichen Geschäftsumsatz veranschlagt. Ohne die Genehmigung und ausdrückliche Bestimmung der königl. Regierung hätte keil junger Apotheker ein fast werthloses und so theuer erkauftes unveriusserliches Gut acquirirt.

Es ist hier nicht der Ort, mit dem II'n. Garbe eine nähere Untersuchung der Ansprüche der Belheiligten zu führen. Den hohen und höchsten Staatsbehörden sind gut motivirte Petitionen in hinreichender Menge eingesandt. Auch sind die betreffenden Klagen und Bittschriften, worin die Ansprüche derselben und die nothwendig aus diesen Verordnungen entstehenden unglüclilichen Folgen der Wahrheit gemäss hervorgehoben sind, ehrerbietigst und ganz unterthänigst an die Stufen des Thrones unsers gerechten und erhabenen Monarchen niedergelegt, woranf die Betheiligten cincr Antwort entgegensehen. Die letztern hoffen diess mit Zuversicht, uı so mehr, da Se. Excellenz der Hr. Geh. Staalsninister E ich horn unter dem 17. März v. J. auf die Petita dic tröstende Aussicht zu einer Modification gegeben, sowie spätere wohlwollende Aeusserungen Sr. Excellenz, die ganze Sachlage einer nochmaligen Prüfung unterwerfen zu wollen, zu den frohesten Exwartungen berechtigt.

Der Verf. geht nun, nachdem er seinc hunstgenossen als die ärgsten Wucherer an den Pranger gestellı hat, zu den Demonstrationen über, wie die noch bestehenden Privilegien als die Institute zur Plusmacherei angesehen werden, um in möglichst kurzer Zeit recht viel zu gewinnen, und erklärt uns, wie verderblich diese Gerechtsame offenbar den für die Neuzeit als unpassend erkannten, mitteralterlichen Zunftwesen angehören, welches auf alle Gewerbe jeden freien Aufschwung hemmenden Druck ausübt. Hr. Garbe fährt also fort : ,die Medicamente werden unter dem Taxpreise verschleudert; ja, durch allerlei Geschenke die Kunden herbeigelocki, um die Augen des Publicums und des künftigen Käufers zu blenden : Absonderliche Schnurrpfeifereien, die in Conditorläden gehörten, oder allenfalls für die Berliner Dintenfabrik einen würdigen Industriezweig abgeben, werden in Glasschränken mit Spiegelwänden aulbewahrt dem Publico in der Apotheke zur Schau gestellt. Die Apotheke wird zum Prunkzimmer umgestaltel, und kein Luxus wird gespart, um die Augen des Publicums zu blenden; ein wehmüthiger Ingrimm, sagt der Vertheidiger, ergreift mich, wenn ich die der Ausübung der nir über alles theuren Apothekerkunst geweihten Răume diesen, derselben ganz fremden Erwerbzweigen geopfert sehe, und mag man meinen jugendlichen Enthusiasmus vornehn belächeln, so behaupte ich dennoch, es stände schlimn um die Kunst, wenn sie nicht mehre gleichgesinnte Gemüther unter ihren Jüngern zählte, denen ihre Wissenschaft höher gilt als eine Kuh, 
welche sie mit Butter und Käse versorgt xum täglichen Brod! - Der Verf. bemerkt noch, wie der Gehalt der Gehülfen jetzt so herabgeJrückt wäre, dass er in vielen Fällen nicht ausreiche, die Bedürfnisse desselben zu bestreiten, und ergeht sich nun noch in weilläufigen Vorschlägen, auf welche Weise sämmtliche Privilegien, als Unheil bringend, aufuheben für nothwendig zu crachten ist, und wie das am Abgrunde stehende und in Nacht und Finsterniss versinkende Apothekerfach wieder gerettet werden kann, dadurch nämlich, dass man in Berlin eine Apotheker-Acadenie mit einem Normal-Laboratorium gründet, woselbst sämmtliche junge Pharmaceuten das Laboriren lernen sollen.

Wir überlassen es den geehrten Lesern darüber zu urtheilen, wie viel Wahres an diesen Rotomontaden ist. Ob der grossartige Gedanke, eine Apothelier-Academie zu errichten und sämmtliche Privilegia als Verderben bringend für nothwendig erachtet wird, überlassen wir der höheru Weisheit der hohen Staatsbehörden. In Bezug auf das jetzt kärglich gezahlte Salair eubbehrt dieser Satz ebenfalls aller Wahrheit. Ein alter glaubwürdiger Principal erzählte mir, dass er während sciner Servirzeit, vor circa 70 Jahren, in einer der ersten Apotheken Berlins nur 30 Rthlr. Salair per Jahr erhalten hälte. Bei den jetzigen gesteigerten Bedürfnissen aber wird jeder l'rincipal gern seinen Verhältnissen und dem Geschüftsumsatz angemessen salariren, so dass ein Genügsamer damit zufrieden ist.

Der jugendliche Verfasser hat uns somit aus dem Schatze seiner reichen Erfahrungen viel Neues berichtet, besonders viele Schmach über seine Kunstgenossen ausgeschüttet, welches für alle ihrem Fache mit Rechtlichkeit ergebenen Collegen eine betrübende Entdeckung genannt werden müsste, wenn nach Hr. Garbe's Beschreibung wirklich der Untergang der Pharmacie so nahe wäre.

Nehmen wir aber den wahren Kern aus der Brochüre zusammen, so stellt sich heraus, dass Neid, besonders aber Eitelkcit, erzeugt durch das Selbstbewusstsein, viel mehr erlernt zu baben und leisten zu können, als von einem brauchbaren Gehülfen verlangt wirl, allein die Triebfeder zum Erscheinen der Schrift gewesen ist. Dass dio hoffnungslose Aussicht desselben, noch nicht selbstständig zu sein, hierbei eine Hauptrolle spieit, liegt ausser Zweifel. Hr. Ga rbe nimmt am Schlusse wehmüthigen Abschied mit den Worten: "Hiermit schliesse ich denn vorläufig meine Wünsche für das Wohl der Pharmacie, übergebe sie dem Publicum, als die Früchte meines langgenährten Kummers und tiefgewurzelter Besorgniss, welche in mancher Nacht den Schlaf von mir gescheucht etc." Unsere Collegen sind sich über den Schlussact noch nicht einig, ob die schlaflosen Nächte und der langgenährte Kummer, sowie auch der den Verfasser ergriffene wehmüthige Ingrimm über die am Rande des Abgrundes schwebende Pharmacie entstanden ist, oder darüber, dass Fortuna dem Verfasser bis jetzt noch keine Concession zugetheilt hat. $\mathrm{Da}$ wahrscheinlich das Letztere der Fall ist, so wünschen wir, dass es demselben gelingen möge, bald im Besitz derselben sein Gemüth zu erheitern, damit die Trauerflagge fernerhin nicht mehr die wahre und richtige Einsicht in so wichtigen Angelegenheiten verfinstere; dann wird es gewiss besser mit der Pharmacie werden!

$$
\text { Nachtrag von Dr. Bley. }
$$

Wenn, wie vielfach vermuthet worden ist, hinter dem Namen des Hrn. Garbe, sich nicht ein anderer hochgestellter Verfasser verbirgt, 
so ist es allerdings eine sehr grosse Anmassung, wenn ein junger Mann, der doch unmöglich reich an Erfahrung sein kann, sich erdreistet, dem Hrn. Minister des Medicinalwesens im preussischen Staate, denn diesem ist die Schrift gewidmet, so vielfach unwahre Angaben als Wahrheit aufutischen und damit Rathschläge zu verbinden, welche in einer schwierigen Sachlage eine grössere Weisheit erfordern, als nach seinem ganzen Auftreten zu urtheilen, dem Hrn. Verfasser inwohnt. Wenn dic Pharmacie in Deutschland Rückschritte machen sollte, so sind diesc lediglich herzuleiten von der vielfach ungünstigen Stellung in welche neuerlichst dieselbe dureh gesetaliche Bestimmungen gerathen, welche statt den wissenschaftlichen Geist zu wecken, denselben unterdrückt. Dass aber bis jetzt die Pharmacie, Gott sei Dank! noch keine Rückschrilte gemacht hat, beweisen sowohl die vorzüglichen Zustände der meisten deutschen Apotheken, als auch der wissenschaftliche Sinn, der, wenn auch nicht alle, doch schr viele jetzige Apotheker noch belebt. Es würde nicht schwer halten zu beweisen, wie schr der Verfasser in seiner übeln Laune, denn so wollen wir schonend seinen aufgeregten $Z$ ustand nur nènen, sich irrt, wenn er meint, dass es keine ausgezeichnete Apotheker neuerer Zeit mehr gäbe. Unser Verein umfasst unter seinen zwölfhundert Mitgliedlern gar manchen, der mit Fug und Recht ausgezeichnet durch geistige und humane Bildung genannt werden darf, aber auch ausserhalb seines Kreises sind viele ganz ausgczeichnete Männer, die der Pharmacie zur Ehre gereichen, wir erinnern hier nur an Winckler, Herberger, Buchner jun., Molr jun., Reinsch, den verewigten Probst u. A. m.

Dass die Apotheker in ihren Laboratorion weniger als sonst chemische Präparate bereiten, ist nicht abzuläugnen, der Grund davon liegt aber nicht in den geringern Fähigkeilen und Kenntnissen, sondern in dem geringen Verbrauche von Chemikalion zur Arzneibereitung, der grossen Reinheit und den billigen Preisen der Präparale, welche die chenischen Fabriken bieten, der Erlaubniss, solche daraus zu beziehen und dem geringen Lohne, welcher den Apothekern gewäbrt wird. Will man das ändern, so stellc man gesetzlich fest, dass die Apotheker alle oder doch die wichtigsten Prïparate selbst bereiten müssen. Aber ungeachtet dass viele Präparate mögen bezogen werden, so giebt es dennoch gar manche Apothekcr, in deren Laboratorien viele chemische Präparate angefertigt werden. J)ass aber eine pharmaceutische Academie und ein grossartiges Laboratorium, in welcher und in welchem vielen jungen Pharmaceuten Gelegenheit zur Ausbildung geboten werden könnte, nützlich sein würde, ist nicht in Abrede zut stellen, da man ohnehin nur erst auf wenigen Universitäten den Pharmaceuten die nöthige Rücksicht gewährt. Will man die Privilegien der Apotheken aufheben, so würde das im Sinne der Gerechtigkeit nicht anders, als gegen vollständige Entschädigung geschehen könuen oder soll, vielleicht im Sinne des Verfassers, Gewalt vor Recht gehen? Wem einyelne Fälle des Wuchers bei. Apothekenverküufen nicht in Alirede zu stellen sind, so müsste, wo diese vorkommen, die Behörde die Genehmigung versagen, dass aber um einzelner Apotheker Habsucht willen der ganze Stand mit Unbill überhäuft werden soll, ist dieses wohl gerecht?

Die Gehalte der Gehülfen sind gegen ehedem sehr gesteigert; dass sie im Ganzen noch sehr gering sind, ist wohl nicht ganz unrichtig, aber auch das ist wahr, dass viele $A$ potheker sich ausser Stande befinden möchten sie zu erhöhen, eben, weil sich ihre Einnahme schon karg genug stellt. Wenn in manchen Apotheken Nebengeschäfte getrie- 
ben werden müssen, so ist das eben ein Beweis, wie wenig lohnend das Apothekergeschäft in vielen Orten sich stellt, dass eben dadurch die Apotheker zur Ergreifung von Nebenerwerbszweigen gezwungen sind, welche sie gern andern überlassen hätten, wenn nicht die Noth sie dazu aufgefordert.

Wenn der Hr. Verfasser seine Vorschläge zur Verbesserung der Pharmacie allein aus dem reinen Motive geschrieben hätte, derselben, also seinem eigenen Stande zu nützen, so musste derselbe alle Uebertreibung meiden und auf dem Wege der Wahrheit bleiben, die er indess frech verletzt hat. Dass aber dem, der so offenbare Lügen in die Welt schreiben kann, als der Verfasser, nicht das Prädicat eines "wahrhaftigen" Mannes gebührt, steht fest und wem die Wahrhaftigkeit abgeht, dem ist auch kein Glauben zu schenken und er kann auch keinen Anspruch machen anf den Titel eines Beförderers des Guten und Nützlichen: denn wer dazu sich der unedeln Mittel, als der Unwahrheit, der Anfeindung seines eigenen Faches bedient, ja als Verlïumder desselben auftritt, also gegen seine eigene Ehre streitet, der hat sich selbst blossgestellt, hat es sich sèlbst beizumessen, wenn ihm statt der vielleicht gehofften Ehre die Verachtung vieler redlicher Männer seines Standes zu Theil wird.

\section{Ceber die Apotheken-Concessions-Angelegenheit; von $\mathrm{Dr}$. M ïller in Emmerich.}

Vor Kurzem sind im hiesigen Regierungsbezirk Düsseldorf zwei Apotheken nach der in der Ministerialverfügung vom 13. August 1842 verüfentlichen Instruction, nachdem zuvor die Erledigung der Concession im Antsblatte angezeigt worden war, übertragen worden.

Es sollen sich zur Erlangung der Concession für eine dieser Apotheken nicht weniger als 50 Candidaten der Pharmacie bei der Regierung zu Düsseldorf gemeldet haben.

Bekanntlich müssen zu den Anträgen an die Regierung jedesmal Stempelbogen angewendet werden, und ebenso werden die Verfügungen der Regierungen auf Stempelbogen ausgefertigt. Der RegierungsHauptkasse, sowie der Postkasse wird durch das eingeleitete Verfahren des Ministerii bei erledigten Concessionen eine neue, bisher noch unbekannt gewesene Quelle des Absatzes an Stempelbogen und des Aufbringens von Portokosten eröffnet, von der wir sowohl als alle Apotheker gewiss herzlich wünschen, dass sie sich wieder verlieren möge.

Die oben gemeldeten 50 Candidaten der Pharmacio haben der Regierungs - Hauptkasse eingebracht :

a) für Stempelbogen zum Antrage an die Regierung à 5 Sgr. der Bogen ............. 8 Thlr. $10 \mathrm{Sgr}$.

b) für Stempelbogen, worauf die Verfügung der Regierung erlassen, a 15 Sgr. .....25

Summa 33 Thlr. 10 Sgr.

Der Postkasse sind dadurch zugeflossen:

a) für Portokosten des Antrags bei der Regierung, sowie für die miteingesandten sämmtlichen Zeugnisse des Candidaten, durchschnittlich à $10 \mathrm{Sgr} . . . \ldots \ldots . .16 \mathrm{Thl}$. $20 \mathrm{Sgr}$.

b) desgleichen für die den Candidaten zugesandten Verfügungen von Seiten der Regierung, sowie für die zurückgesandten Zeugnisse, durchschnittl à $10 \mathrm{Sgr} . .16 \quad, 20$, 
Eine Apotheke hat also zufolge der Ministerialverfugung rom 13. August dem Staate schon $66 \mathrm{Thlr}$. $20 \mathrm{Sgr}$. excl. der Sporteln und der Kosten für den ernannten Apotheker, welcher die Büchsen taxirt, eingebracht.

Es steht zu erwarten, ja man kann es mit Sicherheit behaupten, dass die nämlichen Candidaten und noch cine Portion dabei, welche sich bereits um die Erlangung ciner Concession meldeten, bei der nächsten besten und so bei jeder Gelegenheit wieder kommen, wieder ihre Zeugnisse einsenden, wieder anfragen und wieder dem Staate so viel einbringen, also in dieser Weise dem State sehr viel nützen *).

Nach der Meinung der Kegierungs-Medicinalräthe (wenn man es glauben darf) wäre denselben bei dem neuen Verfahren von $\Lambda$ pothekenverkäufen, indem sie jedem Candidaten antworten müssen, eine sehr lästige, zeitraubende, und wie es uns scheint, eine ganz überflüssige Arbeit aufgedrungen. Es ist schon so viel Schönes ïber diese neue, die innigsten Familienbande zerreissende Ministerialverfügung in den Blättern des Archivs mitgetheilt worden, welche hinlänglich beweisen, dass solche Neuerungen ganz unnöthig geworden. Denn umsonst hat man bisher forschend nach der Ursache einer so plötzlichen Veründerung in unsern schon sehr gedrückten Verhältnissen gefragt. Niemand vermag sie zu finden, und selbst Behörden bleiben auf die Frage: Warum dieses geschehen - stets die Antwort schuldig.

Ich habe nicht unterlassen können, während der Versammlung der Rheinischen Provinzial-Iandstände gleich nach der Eingabe meines Antrages an dieselben, die Mitglieder auf diese selbst von der höchsten Behörde noch unbeantwortet gebliebenen Frage, sowie auf die Vertretung der Pharmacie durch eigene Fachgenossen offentlich aufmerksam zu machen, und namentlich hervorzuheben gesucht, dass auch nicht ein einziger rechtlicher Grund vorhanden sei, welcher diesen Eingriff in das Eigenthum der Apotheker nothwendig gemacht habe und rechtfertigen lsönnte. Wenn es aber wirklich gegründet sein sollte, dass der Staat diese Maassregel zu Gunsten der vielen noch unversorgten Candidaten der Pharmacic genommen, so vermögen wir nur einen Rückschritt darin zu erkennen. Stellt denn das abgelegte Staatsexamen nicht Jeden gleich, und hat nicht ein jeder Candidat das Recht, sobald er durch das abgelegte Examen für fähig befunden, eine Apotheke (wenn er dazu die Mittel besitzt) zu kaufen? Oder ist die Versicherung, welche in der Approbation vom Staate ausgesprochen, dass jeder Candidat das Recht habe, eine Concession zu verlangen, ein blosses linderspiel? Warum noch einmal eine Auswahl treffen unter gleich Berechtigten, besonders von der Regierung, welche die sich bei der Erledigung einer Concession meldenden Candidaten gar nicht kennt und nicht kennen kann? - es sei denn, dass künftig auch Conduitenlisten über das Apothekerpersonal geführt werden müssten.

*) Ebenso wird dem Staate für die Folge anch eine schöne Summe für Gewerbestener von den Aerzten zufliessen, welche sich mit dem Selbstdispensiren beschäftigen werden (welches ohne $Z$ weifel eine nicht kleine Anzahl sein wird), denn es wäre ungerecht vom Staatc, wenn solche Leute davon befreit blieben, da diese unserm Stande unwürdige Steuer stets noch von uns gefondert wird, während man in Russland und Polen aus Achtung für unsern Stand nicht daran denkt, und Holland bei seiner neuen Reform damit umgeht, die Apotheker davon zu befreien.

Arch. d. Pharm. LXXXVII. Bds. 1. Hft. 
Ueber alles dieses hätte man füglich doch von der höchsten Behörde vor Erlass solch drückender Verfügungen eine Belehrung bedurft, da in allen wohlorganisirten Staaten alle Verordnungen, ehe solche zur Anwendung kommen, gehörig geprüft und erst dann eingeführt werden, wenn das Recht und die Billigkeit sie fordern.

Wir haben in unserer gerechten Angelegenheit an die höchste Instanz appeilirt, an die offentliche Meinung - an die Vertreter des Volks - an die Rheinischen Landstände, und was diese darüber gedacht, gesprochen, ist bereits in den Blättern des Archivs niedergelegt, und ist, wie wir erwarten durften, zu unsern Gunsten, obgleich 13 schwache nicht gehörig in die pharmaceutischen Verbältnisse eingeweihte Stimmen sich auf Veranlassung von Männern gegen uns erhoben, welche eine ganz unbegründete Opposition bilden, und Ansichten entwickelten, von denen wir die moralische Ueberzeugung haben, dass sic selbst nicht einverstanden damit sind. Deshalb wollen wir zuversichtlich hoffen, dass des Königs Majestät uns eingedenk seiner gesprochenen königlichen Worte den nöthigen Schutz in dieser unserer gerechten Angelegenheit nicht versagen wolle, und der nächste Landtagsabschied vielen Hunderten von Familien Balsam für die tiefgeschlagene Wunde spenden möge.

Im October 1843.

\section{Ueber Concessions-Angelegenheit. Auszug aus einem Briefe des Hrn. Vicedir. Sehlmeyer in Cöln an Dr. Bley.}

,Zum Schlusse muss ich Ihnen noch erzählen, dass der Hr. Geh. Staatsminister Dr. Eichhor $n$ in diesen Tagen in Cöln war und einer Deputation der Cölner Apotheker eine Audienz zu ertheilen die Ginade hatte. Da ich der Aelteste der erwảhlten Deputirten war, so wurde mir das Amt eines Sprechers zu Theil. Ich bemühte mich, den Herrn Minister auf die Ungerechtigkeit und Härte der neuern gesetzlichen Bestimmungen aufmerksam zu machen, zeigte ihm, wie sehr dieselben unsere Privatinteressen verletzten, ja unsere Vermögenszustände in Frage stellten, und führte ihm als Beleg das Beispiel der hiesigen Heine'schen Apotheke an. Der Herr Minister hörte mich ruhig an, ohne mich zu unterbrechen, und nachdem ich meinem Herzen Luft gemacht und ihn dringend gebeten hatte, doch diese berührten Uebelstände wieder möglichst von uns abzuw enden, erwiederte er eben so freundlich als gnädig, dass er die Apotheker im Staate hochschätze, dass er jhr nützliches Wirken wohl zu würdigen wisse und gern bereit sei, soweit es von ihm abhinge, alles zu thun, um das Fach und seine Genossen auf einem ehrenvollen Standpuncte zu erhatten. Jass ferner der Erbfolge nichts im Wege stehe, und wenn der Sohn eines Apothekers die Qualification besitze, man ihm dic Uebernahme des Geschäfts des Vaters nicht verweigern werde, eben sowenig cinem etwaigen Schwiegersohne. Was indessen die freie Disposition über das Geschäft in Bezug auf den Verkauf beträfe, so würde diese einigen Beschränkungen unterworfen werden, welche spätern Bestimmungen vorbehalten blieben. Schliesslich legte ich ihm noch unsere Angelegenheiten warm ans Herz. Auch in Aachen hat Hr. College Dr. Monheim in gleichem Sinne gesprochen und auf die Nothwendigkeit der Vertretung der Pharmacie hingewiesen. In Düsseldorf, Elberfeld und Münster würde in ähnlichem Sinne unser Nothstand besprochen werden u. s. w.t' 
Der Verein ist den verehrten Herren Collegen Sehlme yer und Dr. Monheim fũr ihre freimüthige Darlegung der bedrängten Verhältnisse unsers Standes dankbar verbunden und wünscht, dass endlich einmal wieder ein Strahl der Hoffnung auf die so unverschuldet ungünstige Lage der deutschen Pharmaceuten fallen möge. Meinerseits gethane Schritte hoffe ich nächstens zur Kenntniss bringen zu können.

Dr. Bley.

Angelegenheit der concessionirten Apotheker und das Selbstdispensiren homöopathischer Aerste.

Ueber beide Angelegenheiten habe ich mir erlaubt, Sr. Excellenz, unsers Vereins gnädigsten Hrn. Protector, dem Hrn. Geh. Staatsminister Dr. Eichhorn in Berlin eine, auf Wahrheit beruhende, freimüthige Vorstellung unterm 8. Septbr. 1843 einzureichen, nit der Fürbitte, ,die Interessen der durch obige Puncte sehr bedrängten Pharmaceuten einer nochmaligen gnädigen Erwägung zu unterwerfen und, wo möglich, Maassregeln der Erleichterung treffen zu lassen!"6

Hierauf ist im Anfange Decembers die nachstehende Antwort Sr. Excellenz erfolgt, welche ich den Herren Mitgliedern des Vereins zur Kenntnissnahme mitzutheilen mich verpflichtet halte, mit dem Ersuchen, daraus auch Ihrerseits zu entnehmen: "wie zu hotfen stehe, dass Se. Excellenz dic Ihm irgend mögliche Erleichterung, soweit solche mit Berücksichtigung der nothwendigen medicinal-polizeilichen Interessen vereinbar ist, in weitere Erwägung ziehen und demnach ausführen lassen werde!

Hiermit dürfte denn die Besprechung dieser Angelegenheit für jetzt geschlossen sein.

Der Oberdirector Dr. Bley.

Erlass des Hrn. Geh. Staatsministers Dr. Eichhorn an den Oberdirector Dr. Bley.

Ew. Wohlgeboren erwiedere ich auf Ihr gefälliges Schreiben vom 8. Sept. d. J. in Betracht der neuern Verordnungen wegen der Rechtsverhälınisse der concessionirten Apotheker, sowie wegen des Selbstdispensirens homooopathischer Arzneien Seitens approbirter Medicinalpersonen, dass ich jederzeit bereit sein werde, den Interessen der Apotheker, soweit solches mit der pflichtmässigen Fürsorge für das allgemeine sanitätspolizeiliche Interesse vereinbar ist, jede mögliche Berücksichtigung zu Theil werden zu lassen. Es sind daher auch die verschiedenen von Ihnen angeregten Puncte bei der Berathung der oben erwähnten Verordnungen nicht unbeachtet geblieben und werden auch bei den fernern Verhandlungen über die pharmaceutischen Angelegenheiten nicht unerwogen gelassen werden. Ich kann indess nicht umhin, Ew. Wohlgeboren darauf aufmerksam zu machen, dass der Apothekerverein vorzugsweise zur Förderung wissenschaftlicher Zwecke und zur innern Bildung des Apothekerwesens ins Leben gerufen ist, das Einmischen desselben in Verwaltungs - Angelegenheiten einzelner Regierungen aber leicht zu Missdeutungen Veranlassung geben konnte, vor welchen er sich im Interesse seines wahren, fruchtbaren Gedeihens zu hüten hat.

Für die gefällige Mittheilung des August- und Septemberhefts des Archivs der Pharmacie, sowie der von Ihnen herausgegebenen Schrift 
„über die Nothwendigkeit der Vertretung der Pharmacie bei den Medicinalbehörden" danke ich Ew. Wohlgeboren verbindlich.

Berlin, den 26. November 1843.

Der Minister der Geistlichen, Unterrichts - und MedicinalAngelegenheiten.

An den Oberdircctor des Apothekervereins

Eichhorn.

in Norddeutschland, Hrn. Dr. L. F. B le y

NG 5867 . Wohlgeb. in Bernburg.

\section{3) Jubelfeier von Harless und Pfaff.}

\section{Harless' Jubelfeier.}

Bonn, den 19. October 1843.

Am 15. Mai 1844 feiert der Geheime Hofrath und Professor Dr. II arless, der ärzllichen Welt so rühmlich beliannt, den Tag, an welchem er vor 50 Jahren sich die medicinische Doctorwürde erwarb. Dieser Tag bietet den Schülern and Freunden des Jubilars die erwünschte Gelegenheit, demselben anch öffentlich die tiefgefühlte Verehrung bezeugen zu können, die er sich durch ein funfzigjühriges Virken und Lehren in unserer gemeinsamen ärztlichen Wissenschaft so allgemein erworben hat. Das in Bonn aus den ärztlichen Freunden und Schülern erwählte Comite hat zwei Vorschläge gemacht, welche die Art dieser Ehrenbezeigung zu verwirklichen bezwecken. Es war der Meinung, aus einzusammelnden Beiträgen 1) ein Slipendium Harlessianum bei der Universität zu stiften, so dass das Andenken des Jubilars auch noch für die fernste Zukunft gesegnet und in Erinnerung erhalten werde, oder 2) ein Weihgeschenk zu bestellen, am Tage des Jubelfestes $z u$ überreichen.

Die hier in Bonn anwesenden Schüler und Freunde des Jubilars wünschen, dass alle entfernten Schüler und Freunde an diesem Werke sich durch irgend eine Gabe betheiligen mögen, damit das Beginnen melir und mehr der Ausdruck einer allgemeinen Theilnahme ärztlicher Kunstgenossen sei. Sie verfehlen deshalb auch nicht Sie, Hr. College, als einen Freund oder Schüler des Jubilars, zur Theilnahme aufzufordern, in ihrem Kreise Beiträgc bei etwa uns nicht bekannten Schülern und Freunden zu sammeln, und zu bitten, das Eingegangene, bis zum 1. Härz 1844, an den Cassirer des Comité, Ilru. Dr. Zartmann in Bonn, einsenden zu wollen. - Die Namen der Geber werden in einem besondern Album der Festgabe hinzugefïgt werden.

\section{Das Comité.}

Dr. J. F. H. Albers. Dr. Brach. Dr. Ernsts. Ir. Halt. Dr. Marquart. Dr. Zartmann.

\section{Pfaff's Doctor - Jubiläum.}

Am 5. October fand zu Kiel die Jubelfeier der vor 50 Jahren stattgefundenen Doctor - Promotion des berühmten Professors der Chomie Pfaff statt. És war mehr ein Fest der ganzen ärztlichen Welt in den deutsehen Landen Dänemarks, als bloss eine Feier der Universität, die dem treuen noch immer wahrhaft jugendlichen Eifer ihres ältesten Hauptes mit der innigsten Theilnahme begegnet. Am Morgen weckten 